\title{
Die Bedeutung der präoperativen Immunhistochemie bei Patientinnen mit Endometriumkarzinomen - welche Parameter sind entscheidend?
}

\section{S. Marnitz ${ }^{1}$}

Angenommen: 29. September 2021 / Online publiziert: 11. November 2021

(c) Der/die Autor(en) 2021

Hintergrund und Fragestellung Die genomische Klassifikation des Endometriumkarzinoms [2] hat uns vor Augen geführt, dass die Zweiteilung in Typ I und Typ II sowie die klinischen Faktoren wie Myometriuminfiltration, Grading, Lymphangiose (L), Hämangiose (V), Tumorgröße und das Alter der Patientin die Biologie des Tumors nicht ausreichend beschreiben. Das Ergebnis sind insuffiziente Risikostratifizierungen (,low risk, intermediate, high intermediate, high risk"), auf deren Basis Therapieentscheidungen für Tausende von Patientinnen getroffen wurden und z.T. auch weiterhin werden. Zahlreiche klinisch bedeutsame Fragestellungen zum Wert der adjuvanten Therapie, wie z.B. perkutane Therapie (EBRT) vs. Brachytherapie (BT); Radiochemotherapie (RCT) vs. Radiotherapie; RCT vs. Chemotherapie + BT etc. [3-12], führten alle zu inkonklusiven Ergebnissen, da die Patientenselektion auf der Basis der oben aufgeführten klassischen Risikoeinteilungen die Biologie der Erkrankung unvollständig beschreibt [13-15]. Statt der klinischen sind nämlich die genomischen Faktoren entscheidend. Die Einteilung in (1) POLE-Mutationen, (2) Tumoren ohne spezielles molekulares Profil (,non specific molecular profile“ [NSMP]), (3) die mikrosatelliteninstabilen Tumoren (MSI = ,mismatch repair deficiency“ [MMRd]) und (4) die p53-mutierten Tumoren hat eine neue, bisher unbekannte Welt eröffnet.

Originalpublikation Vrede SW, van Weelden WJ, Visser NCM, Bulten J, van der Putten LJM, van de Vijver K, Santacana M et al (2021) Immunohistochemical biomarkers are prognostic relevant in addition to the ESMO-ESGO-ESTRO risk classification in endometrial cancer [1].

Prof. Dr. med. S. Marnitz

simone.marnitz-schulze@uk-koeln.de

1 Klinik für Radioonkologie, Cyberknife und Strahlentherapie, Medizinische Fakultät, Universität zu Köln, Kerpener Str. 62, 50937 Köln, Deutschland
Folgerichtig machte sich die PORTEC-Gruppe an die Reanalyse ihrer Studienergebnisse, erhob von den in Studien behandelten Patientinnen dieses molekulare Profil und korrelierte es mit dem onkologischen Ergebnis [16]. Dabei zeigte sich, dass POLE-Mutationen zu einem sehr günstigen onkologischen Outcome passen, NSMP- und MSI-Patientinnen eine intermediäre Prognose aufweisen und p53Patientinnen eine deutlich ungünstigere Gruppe darstellen. Es existieren Schnittmengen mit den klassischen klinischen Prognosefaktoren, aber auch mit vielen Patientinnen, die keiner adjuvanten Therapie bedurft hätten (POLE-Mutationen), und Patientinnen, die p53-Mutationen aufweisen und in der Vergangenheit eher das Risiko einer Untertherapie hatten.

Auf der Basis dieser Daten hat die europäische Leitliniengruppe bereits ihre Risikoeinteilung inklusive der Therapieempfehlungen angepasst [17]. Für die deutsche Leitlinie steht dies noch aus [18]. Da bisher keine LevelI- oder -II-Evidenz vorliegt, sind die logische Konsequenz nun Studien, die das genomische Profil der Patientin zur Entscheidungsgrundlage machen, wie z.B. die laufende PORTEC-4a-Studie (ClinicalTrials.gov Identifier: NCT03469674). Die nachfolgend zu kommentierende Arbeit diskutiert, inwieweit zusätzliche immunhistochemische (IHC) Parameter, wie das Adhäsionsmolekül L1CAM und das in die ESGO/ESTRO-Leitlinien aufgenommene p53 sowie ,alte Bekannte“ wie der Hormonrezeptorstatus, zu einer weiteren Verfeinerung der Prognosegruppen und somit der Therapieempfehlungen beitragen können.

Material und Methoden Die Daten von > 760 Patientinnen aus 10 europäischen Kliniken wurden ausgewertet. Das mittlere Follow-up betrug 5,5 Jahre. 71\% der Patientinnen wurden präoperativ als $\mathrm{G} 1 / 2$ diagnostiziert, $89 \%$ wiesen eine endometrioide Histologie auf.

Ergebnisse Die präoperative Immunhistochemie zeigte p53 abn $_{\text {-Befunde bei }} 112(14,7 \%)$, L1CAM+ bei $79(10,4 \%)$ und einen negativen ER/PR-Status bei 151 (20\%) der Pati- 
Abb. 1 Rezidivfreies Überleben von Patientinnen mit $\mathrm{p} 53_{\mathrm{wt}}$ versus p53 $3_{\mathrm{abn}}$ in entsprechenden Kombinationen mit dem Adhäsionsmolekül L1CAM und dem Rezeptorstatus [1]

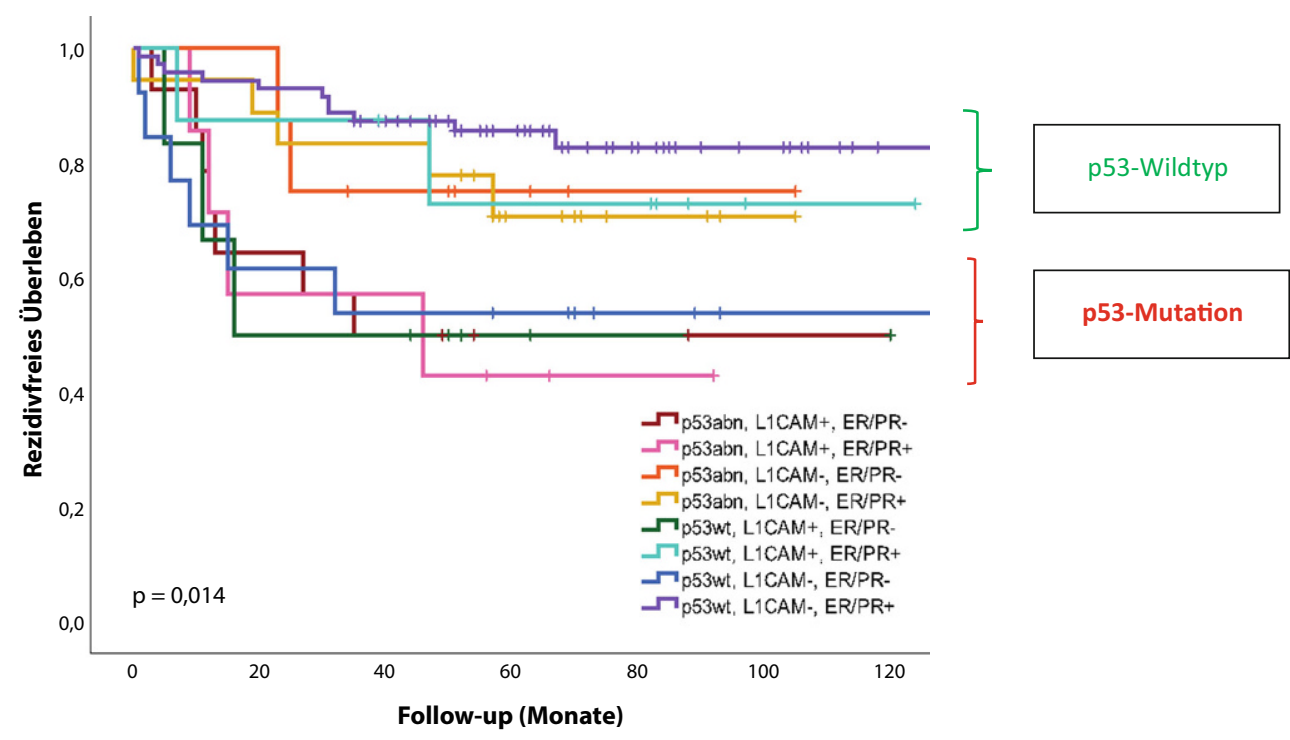

entinnen. Bei 493 (65\%) Patientinnen war eine Lymphadenektomie erfolgt, 53 Patientinnen (11\%) wiesen Lymphknotenmetastasen auf. Die Indikation zur adjuvanten Therapie wurde bei $347(46 \%)$ gestellt. Von diesen 112-mal eine VBT, 104-mal eine EBRT und 93-mal eine VBT+EBRT. Eine Chemotherapie bzw. Radiochemotherapie erhielten 38 bzw. 26 Patientinnen. $102(13,4 \%)$ der 105 (13,8\%) Patientinnen, die ein Rezidiv entwickelten, starben an der Erkrankung. $12 \%$ der hier untersuchten Patientinnen wiesen immunhistochemisch ein abnormes p53 auf; dies gilt als valider Surrogatparameter zur p53-Mutationsuntersuchung. p53 abn erwies sich als wichtigster Prognosefaktor.

Die Studie bestätigt, dass p53 eine Schlüsselstellung einnimmt und der wichtigste Diskriminator zwischen guter/intermediärer Prognose (p53-negative Tumoren) und schlechtem Outcome (p53 $3_{\text {mut }}$ ) ist. Dies ist eindrucksvoll Abb. $1 \mathrm{zu}$ entnehmen. Die zusätzlichen Faktoren wie L1CAM und die guten alten Hormonrezeptoren modulieren lediglich marginal. Dabei beeinflussten L1CAMNegativiät und Hormonrezeptorpositivität das klinische Outcome zum Besseren, L1CAM-Positivität und Hormonrezeptornegativität beeinflussten das Outcome ungünstig.

Schlussfolgerung der Autoren Der Einsatz von präoperativen IHC-Biomarkern hat neben der ESMO-ESGO-ESTRORisikoklassifizierung und neben dem Lymphknotenstatus eine wichtige prognostische Bedeutung. Für die tägliche klinische Praxis könnte die p53/L1CAM/ER/PR-Expression als Indikator für das chirurgische Staging und die Verfeinerung der selektiven adjuvanten Therapie durch Einordnung in die ESMO-ESGO-ESTRO-Risikoklassifizierung dienen [19].

\section{Kommentar}

Die genomische Einteilung hat zu vier Subgruppen geführt, die eine unterschiedliche Prognose anzeigen und sich bezüglich des Ansprechens z.B. auf Immuntherapie deutlich unterscheiden. Faktoren, wie die bekannten Hormonrezeptoren oder das Adhäsionsmolekül L1CAM, haben einen zusätzlichen Einfluss, aber die wichtigste Unterscheidung bietet $\mathrm{p} 53$.

Auch heute kann hier schnell und preiswert schon in jedem Op.-Präparat die Unterscheidung getroffen werden. Die anderen Untersuchungen sind aufwendiger und teurer. An ihnen werden wir in Zukunft aber wohl nicht vorbeikommen, obwohl leider die deutsche Leitlinie aufgrund zeitraubender formaler Prozesse eine der wenigen Leitlinien ist, die hier noch kein Update publiziert haben. Hier sei auf die ESGO/ESMO-Leitlinie [17] verwiesen bzw. auf die NCCN-Leitlinie (Version 3/21; [19]). Die genomische Klassifizierung wird also in Zukunft eine Entscheidungsgrundlage bieten für den Ausschluss einer Hochrisikosituation (p53 $3_{\mathrm{wt}}$ ) bzw. das Vorliegen einer Hochrisikosituation $\left(\mathrm{p} 53_{\mathrm{mut}}\right)$. Dies wird aller Voraussicht nach auch eine wichtige Entscheidungshilfe für die Indikation zur Lymphonodektomie sein, den Einsatz der postoperativen perkutanen statt der Brachytherapie und die Indikation zur Chemotherapie klären.

\section{Fazit}

Bei Vorliegen von POLE-Mutationen handelt es sich wahrscheinlich um Patientinnen, die nicht von einer adjuvanten Therapie profitieren. MSI-Patientinnen haben eine intermediäre Prognose, sprechen aber auf Immuntherapien sehr gut 
an und die Subgruppe der NSMP-Patientinnen bedarf in Zukunft sicher noch einer verfeinerten Subgruppenanalyse. Klinische Studien werden in den nächsten Jahren zeigen, ob der Weg der genomischen Klassifikation bezüglich Prognose und Therapieentscheid der richtige ist.

\section{Simone Marnitz, Köln}

Funding Open Access funding enabled and organized by Projekt DEAL.

Interessenkonflikt S. Marnitz gibt an, dass kein Interessenkonflikt besteht.

Open Access Dieser Artikel wird unter der Creative Commons Namensnennung 4.0 International Lizenz veröffentlicht, welche die Nutzung, Vervielfältigung, Bearbeitung, Verbreitung und Wiedergabe in jeglichem Medium und Format erlaubt, sofern Sie den/die ursprünglichen Autor(en) und die Quelle ordnungsgemäß nennen, einen Link zur Creative Commons Lizenz beifügen und angeben, ob Änderungen vorgenommen wurden.

Die in diesem Artikel enthaltenen Bilder und sonstiges Drittmaterial unterliegen ebenfalls der genannten Creative Commons Lizenz, sofern sich aus der Abbildungslegende nichts anderes ergibt. Sofern das betreffende Material nicht unter der genannten Creative Commons Lizenz steht und die betreffende Handlung nicht nach gesetzlichen Vorschriften erlaubt ist, ist für die oben aufgeführten Weiterverwendungen des Materials die Einwilligung des jeweiligen Rechteinhabers einzuholen.

Weitere Details zur Lizenz entnehmen Sie bitte der Lizenzinformation auf http://creativecommons.org/licenses/by/4.0/deed.de.

\section{Literatur}

1. Vrede SW, van Weelden WJ, Visser NCM, Bulten J, van der Putten LJM, van de Vijver K, Santacana M, Colas E, Gil-Moreno A, Moiola CP et al (2021) Immunohistochemical biomarkers are prognostic relevant in addition to the ESMO-ESGO-ESTRO risk classification in endometrial cancer. Gynecol Oncol 161(3):787-794

2. Cancer Genome Atlas Research N, Kandoth C, Schultz N, Cherniack AD, Akbani R, Liu Y, Shen H, Robertson AG, Pashtan I, Shen $R$ et al (2013) Integrated genomic characterization of endometrial carcinoma. Nature 497(7447):67-73

3. Sorbe BG, Horvath G, Andersson H, Boman K, Lundgren C, Pettersson B (2012) External pelvic and vaginal irradiation versus vaginal irradiation alone as postoperative therapy in medium-risk endometrial carcinoma a prospective, randomized study-quality-oflife analysis. Int J Gynecol Cancer 22(7):1281-1288

4. Sorbe B, Nordstrom B, Maenpaa J, Kuhelj J, Kuhelj D, Okkan S, Delaloye JF, Frankendal B (2009) Intravaginal brachytherapy in FIGO stage I low-risk endometrial cancer: a controlled randomized study. Int J Gynecol Cancer 19(5):873-878

5. Sorbe B, Horvath G, Andersson H, Boman K, Lundgren C, Pettersson B (2012) External pelvic and vaginal irradiation versus vaginal irradiation alone as postoperative therapy in medium-risk endometrial carcinoma - a prospective randomized study. Int J Radiat Oncol 82(3):1249-1255

6. Shaikh T, Churilla TM, Mantia-Smaldone GM, Chu C, Rubin SC, Anderson PR (2016) The role of adjuvant radiation in lymph node positive endometrial adenocarcinoma. Gynecol Oncol 141(3):434-439

7. Randall ME, Filiaci VL, Muss H, Spirtos NM, Mannel RS, Fowler J, Thigpen JT, Benda JA, Gynecologic Oncology Group S (2006) Randomized phase III trial of whole-abdominal irradiation versus doxorubicin and cisplatin chemotherapy in advanced endometrial carcinoma: a Gynecologic Oncology Group Study. J Clin Oncol 24(1):36-44

8. Randall ME, Filiaci V, McMeekin DS, von Gruenigen V, Huang H, Yashar CM, Mannel RS, Kim JW, Salani R, DiSilvestro PA et al (2019) Phase III trial: adjuvant pelvic radiation therapy versus vaginal brachytherapy plus paclitaxel/carboplatin in high-intermediate and high-risk early stage endometrial cancer. J Clin Oncol 37(21):1810-1818

9. Matei D, Filiaci V, Randall ME, Mutch D, Steinhoff MM, DiSilvestro PA, Moxley KM, Kim YM, Powell MA, O’Malley DM et al (2019) Adjuvant chemotherapy plus radiation for locally advanced endometrial cancer. N Engl J Med 380(24):2317-2326

10. Nout RA, Smit VT, Putter H, Jurgenliemk-Schulz IM, Jobsen JJ, Lutgens LC, van der Steen-Banasik EM, Mens JW, Slot A, Kroese MC et al (2010) Vaginal brachytherapy versus pelvic external beam radiotherapy for patients with endometrial cancer of highintermediate risk (PORTEC-2): an open-label, non-inferiority, randomised trial. Lancet 375(9717):816-823

11. Creutzberg CL, Nout RA, Lybeert ML, Warlam-Rodenhuis CC, Jobsen JJ, Mens JW, Lutgens LC, Pras E, van de Poll-Franse LV, van Putten WL et al (2011) Fifteen-year radiotherapy outcomes of the randomized PORTEC-1 trial for endometrial carcinoma. Int J Radiat Oncol Biol Phys 81(4):e631-638

12. de Boer SM, Powell ME, Mileshkin L, Katsaros D, Bessette P, Haie-Meder C, Ottevanger PB, Ledermann JA, Khaw P, Colombo A et al (2018) Adjuvant chemoradiotherapy versus radiotherapy alone for women with high-risk endometrial cancer (PORTEC-3): final results of an international, open-label, multicentre, randomised, phase 3 trial. Lancet Oncol 19(3):295-309

13. Marnitz S, Kohler C, Gharbi N, Kunze S, Jablonska K, Herter J (2018) Evolution of adjuvant treatment in endometrial cancer-no evidence and new questions? Strahlenther Onkol 194(11):965-974. https://doi.org/10.1007/s00066-018-1339-6

14. Marnitz S, Schomig-Markiefka B (2021) The PORTEC-3 trial for high-risk endometrial cancer: impact of molecular classification on prognosis and benefit from adjuvant therapy. Strahlenther Onkol 197(3):266-268

15. Marnitz S, Waltar T, Kohler C, Mustea A, Schomig-Markiefka B (2020) The brave new world of endometrial cancer : future implications for adjuvant treatment decisions. Strahlenther Onkol 196(11):963-972

16. Wortman BG, Nout RA, Bosse T, Creutzberg CL (2019) Selecting adjuvant treatment for Endometrial carcinoma using molecular risk factors. Curr Oncol Rep 21(9):83

17. Concin N, Matias-Guiu X, Vergote I, Cibula D, Mirza MR, Marnitz S, Ledermann J, Bosse T, Chargari C, Fagotti A et al (2021) ESGO/ESTRO/ESP guidelines for the management of patients with endometrial carcinoma. Int J Gynecol Cancer 31(1):12-39

18. S3-Leitlinie zur Diagnostik TuNdPmE: Interdisziplinäre s3-Leitlinie. www.awmfde 2018.

19. National Cancer I Version 03/21. https://www.nccn.org/guidelines/ category_2021. Zugegriffen: 01. Okt. 2021 